\title{
Clinical evaluation of clobetasone butyrate eye drops in episcleritis
}

\author{
D. LLOYD-JONES, A. TOKAREWICZ, AND P. G. WATSON \\ From the Department of Clinical Ophthalmology, Moorfields Eye Hospital, London
}

SUMmaRY Thirty-nine patients took part in a double-blind, between-patient clinical trial to compare clobetasone butyrate, betamethasone phosphate, and placebo eye drops in the treatment of episcleritis. Although from the symptom scores the patients given placebo appeared to do as well as the other patients in the first week of treatment, they did significantly less well after this time. Clobetasone butyrate and betamethasone phosphate eye drops seemed to be equally effective in the treatment of this disease.

Episcleritis is a generally benign, often bilateral inflammatory condition occurring principally in younger adults. The onset is usually acute, presenting with redness of the eye; this is often sectorial and may be accompanied by mild discomfort and occasionally watering and photophobia. Localised episcleral nodules may or may not be present but are of no prognostic significance. In contrast with scleritis, ocular tenderness is absent, and pain, if present, is localised to the eye, rarely radiating to the periorbital region and never producing the severe boring pain characteristic of scleral inflammation.

Since the inflammatory process is confined to the episcleral tissue and superficial episcleral vessels a drop of $10 \%$ phenylephrine will blanche the eye within minutes in contrast to scleritis, in which the deep episcleral vessels remain engorged over the underlying oedematous sclera.

The condition is often self-limiting, but when the symptoms demand treatment topical corticosteroids, either as drops or ointment, are the standard therapy. Their efficacy is accepted, although so far only one double-blind trial has been published. '

The ability of certain topically applied corticosteroids to raise intraocular pressure (IOP) in susceptible individuals limits the usefulness of these potent anti-inflammatory agents. However, clobetasone butyrate eye drops have been found to have less of a propensity to raise intraocular pressure in these sensitive patients. ${ }^{23}$

This trial was carried out to assess the efficacy of clobetasone butyrate eye drops in the treatment of episcleritis.

Correspondence to Mr D. Lloyd-Jones, FRCS, Royal East Sussex Hospital. Hastings TN34 1ER.

\section{Patients and methods}

This was a double-blind, between-patient study extending over a period of 3 weeks. Thirty-nine patients entered the trial, of whom 5 did not return after the initial visit. Patients received either clobetasone butyrate eye drops (12 patients), betamethasone sodium phosphate eye drops (11 patients), or placebo drops (11 patients). The eye drops were administered 4 times a day. Before random allocation to one of the 3 treatment groups patients were stratified according to whether nodules were present and whether they had already received corticosteroid therapy.

Patients were assessed for pain, conjunctival injection, and episcleral injection and scored as 0 absent, 1 slight, 2 moderate, and 3 marked, the latter 2 parameters being assessed for each quadrant of the eye. Nodules were also scored as 0 none, 1 just definite, 2 obvious, 3 confluent. Patients were assessed at the initial visit and after 3, 7, 14, and 21 days of treatment. On each occasion visual acuity and intraocular pressure were measured.

\section{Results}

SCORES

The sources on each visit were summated, and Fig. 1 shows the mean scores. The data have also been analysed by using the scores for episcleral injection alone, but the results were almost identical. The mean scores of the 2 steroid groups were very similar. The scores in the placebo group indicated that the patients improved, the scores being about the same as the other groups after 1 week's treatment. However, 
MEAN SYMPTOM SCORE



Fig. 1 Mean symptom score. Key: $-\times-$ Clobetasone butyrate eye drops $(n=12)$. - - Betamethasone sodium phosphate eve drops $(n=11) .--\square--$ Placebo eve drops $(n=10)$.

after this time the patients appeared to do less well than those receiving the steroid drops.

RELIEF OF SYMPTOMS

Fig. 2 shows the number of patients in whom symptoms were completely resolved (i.e., symptom scores of 0 ) after 3, 7, 14, and 21 days of treatment. Significantly fewer patients were free of symptoms after 21 days' treatment in the placebo group than in the other two groups $\left(p<0 \cdot 05, \chi^{2}\right.$ test $)$.

Six patients in the placebo group had an initial decrease in symptom score (in 2 cases there was a complete relief of symptoms) followed by an increase. This did not occur in any of the patients receiving steroid eye drops.

\section{WITHDRAWALS AND SIDE EFFECTS}

One patient in the placebo group was withdrawn from the trial because the condition was too severe after 3 days of treatment. The scores from this patient were excluded from the calculations. One patient on clobetasone butyrate was withdrawn after 1 week's therapy because of severe staphylococcal lid infection; during the week the episcleritis had resolved. One patient in the betamethasone group discontinued the drops after 3 days because of irritation on instillation; her symptoms had resolved during the 3 days' treatment.

INTRAOCULAR PRESSURE

Changes of less than $6 \mathrm{mmHg}$ over the course of treatment were considered not significant. There was a significant pressure rise in only 2 patients. The largest increase in IOP was observed in one patient in the betamethasone group $-14 \mathrm{mmHg}$ increase over 3 weeks (from 14 to $28 \mathrm{mmHg}$ ). This patient was treated for a subsequent attack of episcleritis with clobetasone butyrate drops for 3 weeks, and the IOP rose by only $6 \mathrm{mmHg}$, from 15 to $21 \mathrm{mmHg}$.

One patient who was treated with clobetasone butyrate only in the left eye had pressure rises in both eyes, $7 \mathrm{mmHg}$ in the right and $9 \mathrm{mmHg}$ in the left. A subsequent 3-week course of clobetasone butyrate to both eyes produced a rise from 15 to $22 \mathrm{mmHg}$ in both eyes. Unfortunately the response to betamethasone eye drops could not be assessed due to nonattendance.

\section{Conclusion}

The symptoms of episcleritis appeared to be resolved more satisfactorily with steroid eye drops than with placebo. In particular there were more relapses

CUMULATIVE NUMBER OF PATIENTS IN WHOM THE SYMPTOMS HAD COMPLETELY RESOLVED



Fig. 2 Cumulative number of patients in whom the symptoms have completely resolved. Key: $-\times-$ Clobetasone butyrate eye drops $(n=12)$. - Betamethasone sodium phosphate eye drops $(n=11)$. $-\square-\square$ - Placebo eye drops $(n=10)$. 
within 3 weeks in patients receiving placebo. Clobetasone butyrate and betamethasone sodium phosphate seem to be equally effective in the treatment of this disease.

We acknowledge the support of Glaxo Group Research Limited. We thank Miss Elizabeth Garner for her help and Miss Julie Scheidegger for secretarial assistance.

\section{References}

1 Watson PG. McKay DAR. Clemett RS. Wilkinson P. Treatment of episcleritis. Br J Ophthalmol 1973; 57: 866-70.

2 Ramsell TG, Bartholomew RS. Walker SR. Clinical evaluation of clobetasone butyrate: a comparative study of its effects in postoperative inflammation and on intraocular pressure. $\mathrm{Br} J$ Ophthalmol 1980; 64: 43-5.

3 Dunne JA. Travers JP. A double-blind clinical trial of topical steroids in anterior uveitis. Br J Ophthalmol 1979; 63: 762-7. 